\title{
Six-Years Disease-Free Survival after Isolated Hypoxic Pelvic Perfusion with Chemofiltration for Advanced Cervical Carcinoma
}

Karl R. Aigner* and Sabine Gailhofer

Medias Klinikum GmbH \& Co KG, Krankenhausstraße 14a, 84489 Burghausen, Germany

\section{Introduction}

Cervical cancer is a highly preventable malignant disease and if detected early has a good prognosis and can be cured by surgery or radiotherapy alone. In western countries about 40,000 women die from advanced cervical cancer per year, in developing countries, however, where early diagnosis is the exception, the death rate from advanced cervical cancer is about six times as much. The treatment of choice is intracavitary brachytherapy, a localized radiotherapy which generates a much higher local dose of irradiation to affect advanced cervical cancer than external beam irradiation. It is so far the only means to induce substantial and long lasting remission induction and pelvic control rates up to $90 \%$ at three years $[1,2]$ due to the extremely high local exposure to radiotherapy. Concomitant chemotherapy with cisplatin reduces the relative risk of death by approximately $50 \%$ [3-7]. With surgery alone, however, in stage I tumors, chances of adequate treatment do not exceed 12\% [8]. Effective locoregional therapy, even in advanced stage IIIa disease not invading the pelvic side wall, in terms of radiochemotherapy may induce downsizing of the tumor burden to surgical resectability. The actual standard of care for downsizing is induction chemotherapy combined with external beam irradiation and intracavitary brachytherapy [2]. Despite highly effective intracavitary brachytherapy large volume tumors account for the majority of cervical cancer deaths [9].

Despite high local efficacy and a minimized risk of relapse, the impact of intracavitary brachytherapy on jeopardized quality of life such as lymph edema of the legs, tissue fibrosis related stenosis or occlusion of ureters requiring stents or kidney fistulas and rectovaginal or vesicovaginal fistulas is evident $[10,11]$.

As long as there is no adequate alternative with less collateral damage to adjacent tissues patients have to cope with what can currently be offered as best medical practice. There have been attempts to figure out whether high dose locoregional chemotherapy might induce comparable local remission induction as localized radiotherapy without causing collateral damage and toxicity [12]. Intra-arterial infusion is the most commonly applied technique, however, local drug exposure can be increased by means of isolation perfusion techniques.

\section{Isolated Pelvic Perfusion}

Drug exposure can best be controlled with isolation perfusion techniques. Isolation of the pelvis is carried out via an arterial and venous femoral access. Both femoral vessels are cannulated with so-called stopflow balloon catheters, which, after injection of chemotherapeutics into the arterial catheter are blocked above the bifurcation of aorta and vena cava. Isolation of the pelvis is achieved by means of pneumatic cuffs around the upper thighs (Figure 1). Isolation and local drug exposure usually is 15 minutes. After deflation of the balloon catheters and the upper thigh pneumatic blocks, chemofiltration is started in order to reduce systemic drug exposure. Reduction of systemic peak drug levels prevents or reduces systemic toxicity and side-effects. At the given drug dosages damage of pelvic organs never happened and quality of life is usually undisturbed.

\section{Case Report}

A 58 year old female presented with progressive stage IVa cervical cancer with tumor invasion of the bladder, lymphnodes and both parametria. Staging laparotomy had revealed a non-mobile uterus with a bulky cervical tumor invading the bladder. Regional lymphnodes between $2 \mathrm{~cm}$ and $3 \mathrm{~cm}$ in diameter were removed. Systemic chemotherapy with carboplatin and paclitaxel was started postoperatively. After three cycles, that were not well tolerated, progressive disease was noted in MRI.

After discontinuation of systemic chemotherapy the patient received four courses of isolated pelvic perfusion with cisplatinum (50 $\mathrm{mg})$, adriamycin $(30 \mathrm{mg})$ and mitomycin $(30 \mathrm{mg} / 20 \mathrm{mg})$ and subsequent chemofiltration for systemic detoxification. Isolated perfusions are usually performed in four weeks intervals. After the second perfusion control-MRI revealed a $70 \%$ reduction of the tumor volume. At the time of hysterectomy there was a normal sized uterus without palpable regional lymphnodes and a small residual tumor at the cervix of about $1 \mathrm{~cm}$ in diameter which appeared macroscopically completely necrotic (Figure 2). In the resected specimen pathologic complete remission was noted without evidence of residual tumor tissue.

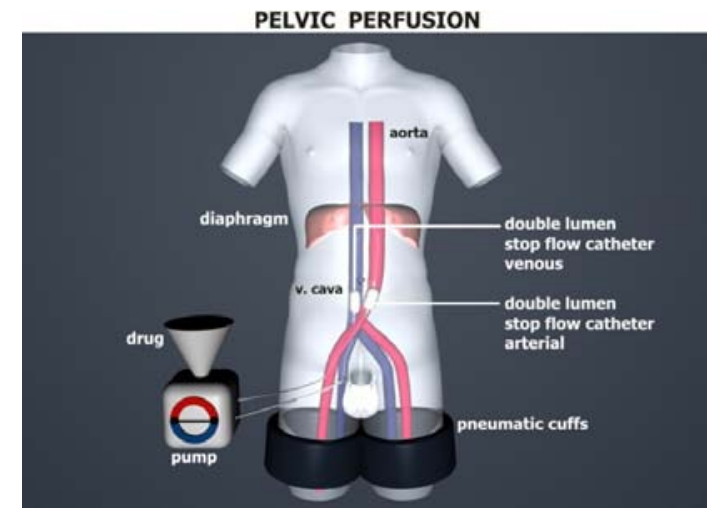

Figure 1: Balloons of three channel stopflow catheter blocked above the bifurcation of aorta and vena cava for isolation of the pelvis, the upper thighs are blocked with pneumatic cuffs, chemofiltration is stared after deflating balloon catheters and pneumatic cuffs.

*Corresponding author: Karl R. Aigner, Medias Klinikum GmbH \& Co KG, Krankenhausstraße 14a, 84489 Burghausen, Germany, Tel: +49 (0) 8677/9160-0; Fax: +49 (0) 8677/9160-120; E-mail: info@prof-aigner.de

Received May 15, 2012; Accepted June 05, 2012; Published June 08, 2012

Citation: Aigner KR, Gailhofer S (2012) Six-Years Disease-Free Survival after Isolated Hypoxic Pelvic Perfusion with Chemofiltration for Advanced Cervical Carcinoma. J Nucl Med Radiat Ther S2:007. doi:10.4172/2155-9619.S2-007

Copyright: (C) 2012 Aigner KR, et al. This is an open-access article distributed under the terms of the Creative Commons Attribution License, which permits unrestricted use, distribution, and reproduction in any medium, provided the original author and source are credited. 


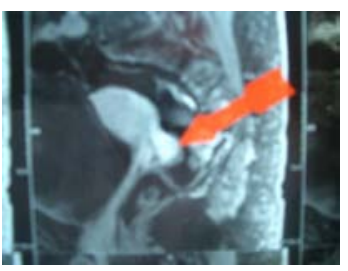

$2 a$

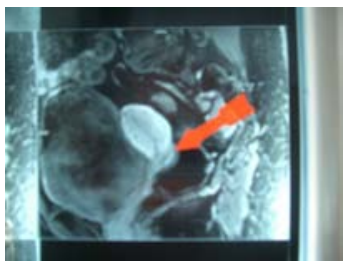

$2 b$
Figure 2a and $\mathbf{2 b}$ : Downsizing of cervical cancer after four courses of isolated pelvic perfusion, inducing pathological complete remission.

An adjuvant cycle of isolated pelvic perfusion was performed six days after hysterectomy. During all five cycles of isolated pelvic perfusion the patient had an undisturbed quality of life without toxic side effects from therapy. During a disease and symptom-free followup time of six and a half years up to date she did not change her lifestyle in business or private life and was active in sports.

\section{Discussion}

Intracavitary brachytherapy with its unique tumoricidal effect from high dose local irradiation exposure is the backbone of guidelines in therapy of cervical cancer and so far in long term pelvic control between 85 and $90 \%$ in combination with platin based chemotherapy has not been surpassed by any other modality. This will remain unchanged as long as there is no comparable equally effective alternative treatment. The only drop bitterness in these apparently perfectly designed guidelines for treatment of advanced cervical cancer are the frequently exorbitant long-term side- effects. The question is whether achieving prolonged survival can justify extreme and often mutilating toxicity. As more and more acute and chronic treatment related psychosocial and physical distress and disfunction are identified, methods are desperately needed to reduce these adverse effects [2]. If dose intense radiotherapy could be completely or at least partially replaced by a less toxic but equally effective localized therapy, avoiding irreversible side effects, patients would certainly benefit tremendously. This underscores the need of an equally potent but less toxic alternative.

Unfortunately regional chemotherapy which might be an option is not sufficiently investigated in extensive studies [13-16]. However, small sample studies so far, report promising and absolutely comparable results in terms of response rates and survival. Until now, intraarterial chemotherapy has not been performed in multicenter studies with uniform protocols, but, regardless of which mode of application has been chosen, be it intra-arterial infusion alone, high dose intraarterial infusion with chemofiltration or isolated perfusion with chemofiltration, results have been remarkable, and it is noteworthy that intra-arterial infusion of pelvic arteries may induce complete remission duration of about 2 years and more without causing any relevant toxicity. Regional chemotherapy creates much higher local drug exposure at low toxicity than does systemic chemotherapy. So far there are only a few studies with few patients but interesting results, addressing this concept.

In a trial comprising 12 patients in clinical stages I to IIb with tumors beyond $4 \mathrm{~cm}$ in diameter intra-arterial infusion therapy through the uterine artery in 7 out of 12 cases induced a tumor mass reduction of more than $50 \%$ of the initial volume after two courses of therapy [13]. In a second study, where intra-arterial infusion chemotherapy with cisplatin, adriamycin and melphalan was administered via both internal iliac arteries, a remission induction of $65 \%$ was achieved, $8.3 \%$ (4/48 patients) of which were complete remissions and the rest were partial remissions. In two cases of complete remission this could be confirmed histologically [14]. In another trial of intra-arterial infusion of cisplatin via hypogastric arteries in 25 patients with advanced $(14 / 25)$ or recurrent $(11 / 25)$ cervical cancer $40 \mathrm{mg}$ of cisplatin was infused over 60 minutes on each side. $78 \%$ of the patients showed a substantial response and radical hysterectomy, lymphadenectomy and external beam irradiation was performed thereafter. In patients with relapsed tumors, the overall response rate was $36 \%$. Pain relief was obtained in all patients. After a mean follow up of 23 months no pelvic recurrence was noted [15]. In another study on 23 untreated patients with stages IIIa to IVa cervical cancer intraarterial high dose cisplatin infusion alone or a combination of cisplatin, mitomycin, bleomycin and 5-FU was combined with chemofiltration for detoxification. In 18 patients the tumor could be downstaged to resectability within two courses of therapy. Interestingly two weeks after the second treatment the median volumetric tumor reduction was $76 \%$ at a tumor response of $87 \%$. Histologic response was $96 \%$ while the tumor downstaging reached $83 \%$. The curative surgery rate achieved was $89 \%$ with a 5 -year progression-free survival of $47 \%$ and a 5 -year survival rate of $74 \%$ [16]. These results are absolutely comparable with data from conventional radiochemotherapy.

Considering these data of locoregional chemotherapy and intracavitary brachytherapy compared to systemic chemotherapy and external beam irradiation it seems obvious that an increase of local exposition, irrespective of which therapeutic modality is applied, leads to enhanced local efficacy. It is also of frontline importance that regional induction chemotherapy for cervical cancer - provided that from the technical point of view sufficient local drug exposure is generated induces immediate tumor shrinkage from the very beginning, at least after one or two therapeutic courses [13-16]. Downsizing of locally advanced cancers to operability is observed in the vast majority of cases. This makes intra-arterial induction chemotherapy seem to be comparable to intracavitary brachytherapy, however, with the advantage, provided that identical efficacy can be documented in larger studies, that there is as much as no collateral damage to adjacent tissues, maintaining or improving the patients quality of life. Therefore a prospective controlled study with the endpoints time to progression, overall survival and quality of life comparing brachytherapy and intraarterial chemotherapy could provide important insight and is urgently needed.

\section{References}

1. Lertsanguansinchai $P$, Lertbutsayanukul $C$, Shotelersuk K, Khorprasert $C$ Rojpornpradit $\mathrm{P}$, et al. (2004) Phase III randomized trail comparing LDR and HDR brachytherapy in treatment of cervical carcinoma. Int J Radiat Oncol Biol Phys 59: 1424-1431.

2. Monk BJ, Tewari KS, Koh WJ (2007) Multimodality therapy for locally advanced cervical carcinoma: state of the art and future directions. J Clin Oncol 25: 2952 2965.

3. Rose PG, Bundy BN, Watkins EB, Thigpen JT, Deppe G, et al. (1999) Concurrent cisplatin-based radiotherapy and chemotherapy for locally advanced cervical cancer. N Engl J Med 340: 1144-1153.

4. Peters WA 3rd, Liu PY, Barrett RJ 2nd, Stock RJ, Monk BJ, et al. (2000) Concurrent chemotherapy and pelvic radiation therapy compared with pelvic radiation therapy alone as adjuvant therapy after radical surgery in high-risk early-stage cancer of the cervix. J Clin Oncol 18: 1606-1613.

5. Keys HM, Bundy BN, Stehman FB, Muderspach LI, Chafe WE, et al. (1999) Cisplatin, radiation and adjuvant hysterectomy compared with radiation and adjuvant hysterectomy for bulky stage IB cervical carcinoma. N Engl J Med 340: 1154-1161.

6. Morris M, Eifel PJ, Lu J, Grigsby PW, Levenback C, et al. (1999) Pelvic radiation 
Citation: Aigner KR, Gailhofer S (2012) Six-Years Disease-Free Survival after Isolated Hypoxic Pelvic Perfusion with Chemofiltration for Advanced Cervical Carcinoma. J Nucl Med Radiat Ther S2:007. doi:10.4172/2155-9619.S2-007

with concurrent chemotherapy compared with pelvic and para-aortic radiation for high-risk cervical cancer: N Engl J Med 340: 1137-1143.

7. Eifel PJ, Winter K, Morris M, Levenback C, Grigsby PW, et al. (2004) Pelvic irradiation with concurrent chemotherapy versus pelvic and para-aortic irradiation for high-risk cervical cancer: An update of Radiation Therapy Oncology Group trial (RTOG) 90-01. J Clin Oncol 22: 872-880.

8. Yessaian A, Magistris A, Burger RA, Monk BJ (2004) Radical hysterectomy followed by tailored postoperative therapy in the treatment of stage IB2 cervical cancer: Feasibility and indications for adjuvant therapy. Gynecol Oncol 94: 6166.

9. Monk BJ, Tewari KS (2007) Invasive cervical cancer: in DiSaia PJ, Creasman WT Clinical Gynecologic Oncology (edn 7) Mosby Publishers, Philadelphia.

10. Vistad I, Fosså SD, Dahl AA (2006) A critical review of patient-rated quality of life studies of long-term survivors of cervical cancer: Gynecol Oncol 102: 563-573.

11. Wenzel L, DeAlba I, Habbal R, Kluhsman BC, Fairclough D, et al. (2005) Quality of life in long-term cervical cancer survivors. Gynecol Oncol 97: 310-317.
12. Stephens FO, (1988) Why use regional chemotherapy? Principles and Pharmacokinetics. Reg Cancer Treat 1: 4 -10.

13. Villena-Heinsen C, Mink D, Lung-Kurt S, et al. (1994) Preoperative intraarterial chemotherapy for bulky cervical carcinoma in stage lb - Ilb. Reg Cancer Treat 1: 17-21.

14. Scarabelli C, Zarrelli A, Gallo A, et al. (1994) Pelvic recurrences in cervica cancer: multimodal treatment with sequential intra-arterial chemotherapy and surgery. Reg Cancer Treat 1: 12-16.

15. de Dycker RP (1994) Pelvic arterial chemotherapy in cervical cancer. Reg Cancer Treat 7: 43-46.

16. Motoyama S, Hamana S, Ku Y, Laoag-Fernandez JB, Deguchi M, et al. (2004) Neoadjuvant high-dose intraarterial infusion chemotherapy under percutaneous pelvic perfusion with extracorporeal chemofiltration in patients with stages IIlaIVa cervical cancer. Gynecol Oncol 95: 576-582.
This article was originally published in a special issue, Surgical oncology: Clinical Importance handled by Editor(s). Dr. Liqiang Zhang, Arizona State University, USA; Dr. Salomone Di Saverio, Surgery and Trauma Surgery Unit, Italy 